\title{
Preliminary Incidence and Trends of Infections with Pathogens Transmitted Commonly Through Food — Foodborne Diseases Active Surveillance Network, 10 U.S. Sites, 2006-2017
}

\begin{abstract}
Ellyn P. Marder, $\mathrm{MPH}^{1}$; Patricia M. Griffin, MD¹; Paul R. Cieslak, MD²; John Dunn, DVM ${ }^{3}$; Sharon Hurd, MPH ${ }^{4}$; Rachel Jervis, MPH ${ }^{5}$; Sarah Lathrop, $\mathrm{PhD}^{6}$; Alison Muse, $\mathrm{MPH}^{7}$; Patricia Ryan, MS ${ }^{8}$; Kirk Smith, DVM 9 ; Melissa Tobin-D’Angelo, MD ${ }^{10}$; Duc J. Vugia, MD ${ }^{11}$; Kristin G. Holt, DVM ${ }^{12}$; Beverly J. Wolpert, $\mathrm{PhD}^{13}$; Robert Tauxe, $\mathrm{MD}^{1}$; Aimee L. Geissler, $\mathrm{PhD}^{1}$
\end{abstract}

Despite ongoing food safety measures in the United States, foodborne illness continues to be a substantial health burden. The 10 U.S. sites of the Foodborne Diseases Active Surveillance Network (FoodNet)* monitor cases of laboratory-diagnosed infections caused by nine pathogens transmitted commonly through food. This report summarizes preliminary 2017 data and describes changes in incidence since 2006. In 2017, FoodNet reported 24,484 infections, 5,677 hospitalizations, and 122 deaths. Compared with 2014-2016, the 2017 incidence of infections with Campylobacter, Listeria, non-O157 Shiga toxin-producing Escherichia coli (STEC), Yersinia, Vibrio, and Cyclospora increased. The increased incidences of pathogens for which testing was previously limited might have resulted from the increased use and sensitivity of cultureindependent diagnostic tests (CIDTs), which can improve incidence estimates (1). Compared with 2006-2008, the 2017 incidence of infections with Salmonella serotypes Typhimurium and Heidelberg decreased, and the incidence of serotypes Javiana, Infantis, and Thompson increased. New regulatory requirements that include enhanced testing of poultry products for Salmonella ${ }^{\dagger}$ might have contributed to the decreases. The incidence of STEC O157 infections during 2017 also decreased compared with 2006-2008, which parallels reductions in isolations from ground beef. ${ }^{\S}$ The declines in two Salmonella serotypes and STEC O157 infections provide supportive evidence that targeted control measures are effective. The marked increases in infections caused by some Salmonella serotypes provide an opportunity to investigate food and nonfood sources of infection and to design specific interventions.

FoodNet conducts active, population-based surveillance for laboratory-diagnosed infections caused by Campylobacter, Cryptosporidium, Cyclospora, Listeria, Salmonella, STEC,

\footnotetext{
*Connecticut, Georgia, Maryland, Minnesota, New Mexico, Oregon, Tennessee, and selected counties in California, Colorado, and New York https://www.cdc. gov/foodnet.

$\dagger$ https://www.fsis.usda.gov/wps/portal/fsis/topics/food-safety-education/getanswers/food-safety-fact-sheets/foodborne-illness-and-disease/salmonella/ sap-two-year and https://www.fsis.usda.gov/wps/wcm/connect/fb8c866a-a9b74b0d-81c9-0f190c4a8d4d/2011-0012F.htm?MOD=AJPERES.

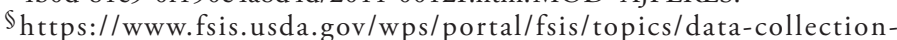
and-reports/microbiology/ec/e-coli-o 157 h7-year-to-date/ ecoli-o157-raw-beef-testing-data-ytd.
}

Shigella, Vibrio, and Yersinia in 10 sites that account for approximately $15 \%$ of the U.S. population (an estimated 49 million persons in 2016). FoodNet is a collaboration among CDC, 10 state health departments, the U.S. Department of Agriculture's Food Safety and Inspection Service (USDA-FSIS), and the Food and Drug Administration (FDA). Laboratory-diagnosed bacterial infections are defined as isolation of bacteria from a clinical specimen by culture or detection by a CIDT. CIDTs detect bacterial antigens, nucleic acid sequences, or, for STEC, Shiga toxin or Shiga toxin genes. 9 A CIDT-positive-only bacterial infection is a positive CIDT result without culture confirmation. Listeria cases are defined as isolation of L. monocytogenes or detection by a CIDT from a normally sterile site or from placental or fetal tissue in the instance of miscarriage or stillbirth. Laboratory-diagnosed parasitic infections are defined as detection of the parasite from a clinical specimen. Hospitalizations and deaths within 7 days of specimen collection are attributed to the infection. Surveillance for physiciandiagnosed postdiarrheal hemolytic uremic syndrome (HUS) is conducted through a network of nephrologists and infection preventionists and hospital discharge data review. This report includes pediatric HUS cases identified during 2016, the most recent year for which data are available.

Incidence per 100,000 population was calculated by dividing the number of infections in 2017 by the U.S. Census estimates of the surveillance area population for 2016. Incidence measures include all laboratory-diagnosed infections reported. A negative binomial model with $95 \%$ confidence intervals (CIs) was used to estimate change in incidence during 2017 compared with that during 2014-2016 and 2006-2008. Because of large changes in testing practices since 2006, incidence comparisons with 2006-2008 used only culture-confirmed bacterial infections, and comparisons with 2014-2016 used culture-confirmed and CIDT-positive-only cases combined. For HUS, 2016 incidence was compared with that during 2013-2015.

\footnotetext{
9 For Shiga toxin-producing Escherichia coli, only CIDT reports that were positive at a state public health laboratory were counted.
} 


\section{Cases of Infection, Incidence, and Trends}

During 2017, FoodNet identified 24,484 cases of infection, 5,677 hospitalizations, and 122 deaths. The incidence of infection per 100,000 population was highest for Campylobacter (19.2) and Salmonella (16.0), followed by Shigella (4.3), STEC (4.2), ${ }^{* *}$ Cryptosporidium (3.7), Yersinia (1.0), Vibrio (0.7), Listeria (0.3), and Cyclospora (0.3) (Table 1). The percentage of CIDT-positive-only infections, including those that were culture-negative and those not tested by culture, were Yersinia (51\%), Campylobacter (36\%), Shigella (31\%), Vibrio (29\%), STEC (27\%), Salmonella (9\%), and Listeria (1\%) (Figure). Compared with incidence during 2014-2016, the 2017 incidence was significantly higher for Cyclospora (489\% increase), Yersinia (166\% increase), Vibrio (54\% increase), STEC (28\% increase), Listeria (26\% increase), and Campylobacter (10\% increase) (Table 1). Bacterial infections diagnosed by CIDT increased $96 \%$ overall (range $=34 \%-700 \%$ per pathogen) in 2017 compared with those diagnosed during 2014-2016. Reflex culture ${ }^{\dagger \dagger}$ was attempted on $71 \%$ of CIDT-positive specimens, ranging from $63 \%$ for Campylobacter to $100 \%$ for Listeria (Figure). Among specimens on which a reflex culture was performed, the percentage of positive cultures ranged from $38 \%$ for Vibrio to $90 \%$ for Salmonella.

Among 6,373 (89\%) fully serotyped Salmonella isolates, the five most common were Enteritidis (incidence $=2.6$ per 100,000), Typhimurium (1.4), Newport (1.3), Javiana (1.1), and the monophasic variant of Typhimurium, I 4,[5],12:i:- (0.9) (Table 2). Among the 13 most common serotypes, the incidence for Heidelberg in 2017 was 65\% lower than during 2006-2008 and 38\% lower than during 2014-2016 (Table 2). It was also significantly lower for Typhimurium for both periods ( $42 \%$ and 14\%, respectively).

Among 1,473 STEC isolates tested for the O157 antigen, $413(28 \%)$ were determined to be O157. Among the 766 non-O157 STEC isolates with serogroup determined, the most common were O26 (29\%), O103 (26\%), and O111 (18\%). During 2017, the incidence of non-O157 STEC significantly increased 25\% (95\% CI $=9-44)$ compared with that during 2014-2016; incidence of STEC O157 was unchanged. However, compared with 2006-2008, the incidence of STEC O157 was significantly lower (35\% decrease; 95\% CI $=21-46$ ).

FoodNet identified 57 cases of HUS in children (incidence $=0.51$ per 100,000$)$ during 2016; $35(61 \%)$ occurred among children aged $<5$ years (incidence $=1.18$ per 100,000 ). The incidence during 2016 compared with that during

\footnotetext{
** Excludes Shiga toxin-positive-only reports from clinical laboratories that were Shiga toxin-negative at a public health laboratory $(n=518)$.

$\dagger \dagger$ Culturing of a specimen with a positive CIDT result.
}

TABLE 1. Incidence of bacterial and parasitic infections in 2017 and percentage change compared with 2014-2016 average annual incidence, by pathogen - FoodNet sites, ${ }^{*}$ 2014-2017 ${ }^{\dagger}$

\begin{tabular}{|c|c|c|c|c|}
\hline \multirow[b]{2}{*}{ Pathogen } & \multicolumn{2}{|c|}{2017} & \multicolumn{2}{|c|}{2017 versus 2014-2016 } \\
\hline & $\begin{array}{l}\text { No. of } \\
\text { cases }\end{array}$ & $\begin{array}{l}\text { Incidence } \\
\text { rate }^{\S}\end{array}$ & $\%$ Change $^{q}$ & (95\% Cl) \\
\hline \multicolumn{5}{|l|}{ Bacteria } \\
\hline Campylobacter & 9,421 & 19.1 & 10 & (2 to 18 ) \\
\hline Salmonella & 7,895 & 16.0 & -5 & (-11 to 1$)$ \\
\hline Shigella & 2,132 & 4.3 & -3 & $(-25$ to 25$)$ \\
\hline $\begin{array}{l}\text { Shiga toxin-producing } \\
\text { E. coli*** }\end{array}$ & 2,050 & 4.2 & 28 & (9 to 50 ) \\
\hline Yersinia & 489 & 1.0 & 166 & (113 to 234$)$ \\
\hline Vibrio & 340 & 0.7 & 54 & (26 to 87 ) \\
\hline Listeria & 158 & 0.3 & 26 & ( 2 to 55 ) \\
\hline \multicolumn{5}{|l|}{ Parasites } \\
\hline Cryptosporidium & 1,836 & 3.7 & 10 & (-16 to 42$)$ \\
\hline Cyclospora & 163 & 0.3 & 489 & ( 253 to 883 ) \\
\hline
\end{tabular}

Abbreviations: $\mathrm{Cl}=$ confidence interval; FoodNet $=C D C$ 's Foodborne Diseases Active Surveillance Network.

* Connecticut, Georgia, Maryland, Minnesota, New Mexico, Oregon, Tennessee, and selected counties in California, Colorado, and New York.

${ }^{\dagger}$ Data for 2017 are preliminary.

$\S$ Per 100,000 population.

I Percentage change reported as increase or decrease.

** For Shiga toxin-producing E. coli, all serogroups were combined because it is not possible to distinguish between serogroups using culture-independent diagnostic tests. Reports that were only Shiga toxin-positive from clinical laboratories and were Shiga toxin-negative at a public health laboratory were excluded $(n=518)$. When these were included, the incidence rate was 5.2 , which was a $57 \%$ increase $(\mathrm{Cl}=33 \%$ to $85 \%)$.

2013-2015 was not significantly different among all children or those aged $<5$ years. The incidence among children aged $<5$ years significantly decreased 36\% $(95 \% \mathrm{CI}=8-55)$ in 2016 compared with 2006-2008.

\section{Discussion}

Clinical laboratories are steadily increasing the use of CIDTs, particularly DNA-based syndrome panels, to diagnose enteric pathogens (2). Previously, routine stool tests typically only included methods for identifying Salmonella, Campylobacter, Shigella, and STEC O157 (3). CIDTs benefit public health by identifying illnesses caused by pathogens not captured routinely by older methods, revealing more accurate incidence estimates for some pathogens. For example, most laboratories required a specific request to test for Cyclospora. Because use of panel tests has risen, routine tests more often include Cyclospora as well as Yersinia, Vibrio, and non-O157 STEC. The increased incidence of these infections in 2017 was most likely driven by the increased use of CIDTs.

Although the number of Salmonella infections with CIDTpositive results increased $176 \%$ during 2017 compared with 2014-2016, the overall percentage without culture confirmation remained relatively low (9\%) because of the high frequency and success of reflex culture, which is necessary for subtyping. Infections caused by serotypes Typhimurium 
FIGURE. Number of infections diagnosed by culture or culture-independent diagnostic tests, by pathogen, year, and culture status — FoodNet sites, ${ }^{*}$ 2014-2017

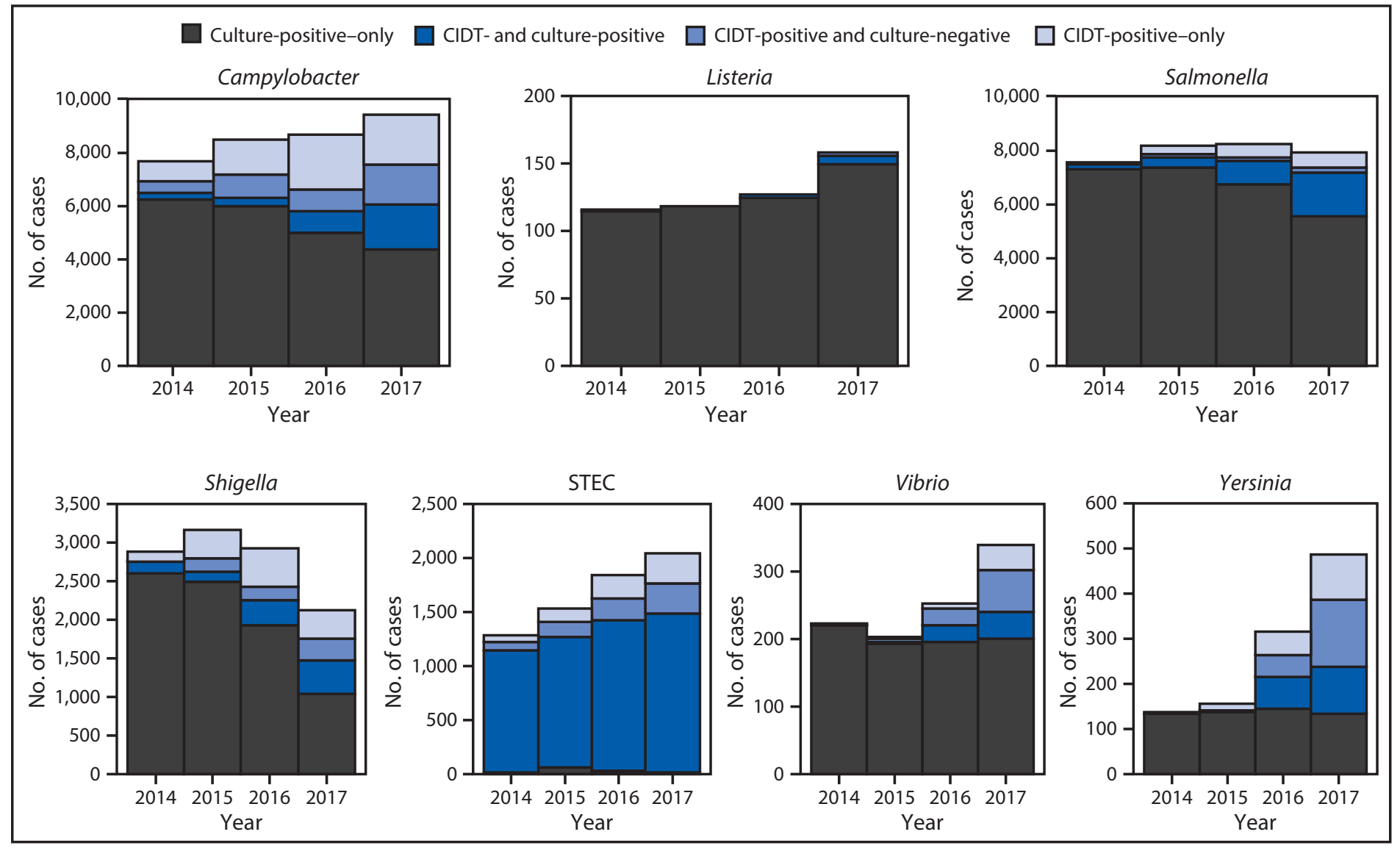

Abbreviations: CIDT = culture-independent diagnostic test; FoodNet = CDC's Foodborne Diseases Active Surveillance Network; STEC $=$ Shiga toxin-producing Escherichia coli.

* Connecticut, Georgia, Maryland, Minnesota, New Mexico, Oregon, Tennessee, and selected counties in California, Colorado, and New York.

+ Data for 2017 are preliminary.

$\S$ For STEC, all serogroups were combined, as it is impossible to distinguish between serogroups using CIDTs. Shiga toxin-positive-only reports from clinical laboratories that were Shiga toxin-negative at a public health laboratory were excluded $(n=518)$.

(including I 4,[5],12:i:-) and Heidelberg have decreased considerably over the past 10 years. These declines mirror decreases in broiler chicken samples that yielded Salmonella and, specifically, serotype Heidelberg (USDA-FSIS, unpublished data). These declines might be partly because of industry measures to vaccinate poultry flocks against these serotypes (4) as well as implementation of measures by USDA-FSIS to decrease Salmonella in poultry and beef products.

Despite these decreases, the overall incidence of Salmonella has not substantially declined since 2014-2016, partly because infections with some serotypes have increased. In particular, infections caused by serotypes Javiana, Thompson, and Infantis each increased approximately 50\% compared with 2006-2008. Like most serotypes, these have been linked to both food and other exposures, including animal contact (5). Thus, some of these infections are likely attributable to nonfood exposures. USDA-FSIS also noted an increase of $>50 \%$ in the percentage of broiler chicken samples that yielded Infantis from 2006 to 2017 (USDA-FSIS, unpublished data).

The decreasing availability of STEC serogroup information, attributable to CIDTs, makes interpretation of trends difficult. However, the decreased incidence of HUS among young children during 2016 compared with that during 2006-2008 provides evidence that supports the finding of a decline in STEC O157 infections because most HUS cases are caused by STEC O157 (6). This decline also mirrors declines in STEC $\mathrm{O} 157$ in ground beef during the same period.

CIDTs pose challenges to public health when reflex culture is not performed. Without isolates, public health laboratories are unable to subtype pathogens, determine antimicrobial susceptibility, and detect outbreaks. Reflex culture recovery rates vary, which could be attributed to false positives, low numbers of bacteria, storage or transport problems, or insensitive culture techniques $(7,8)$. Furthermore, CIDTs vary in sensitivity and 
Morbidity and Mortality Weekly Report

TABLE 2. Incidence of infection of the top 13 Salmonella serotypes in 2017 compared with 2006-2008 and 2014-2016 average annual incidence, by pathogen - FoodNet sites, ${ }^{*} 2006-2017^{\dagger}$

\begin{tabular}{|c|c|c|c|c|c|}
\hline \multirow[b]{2}{*}{ Serotype } & \multirow{2}{*}{$\frac{2017}{\text { Incidence rate }^{\S}}$} & \multicolumn{2}{|c|}{2017 versus 2006-2008 } & \multicolumn{2}{|c|}{2017 versus 2014-2016 } \\
\hline & & $\%$ Change & $(95 \% \mathrm{Cl})$ & $\%$ Change & $(95 \% \mathrm{Cl})$ \\
\hline Enteritidis & 2.6 & 3 & $(-11$ to 20$)$ & -8 & $(-21$ to 7$)$ \\
\hline Typhimurium** & 1.4 & -42 & $(-48$ to -34$)$ & -14 & $(-24$ to -2$)$ \\
\hline Newport & 1.3 & -5 & $(-22$ to 16$)$ & -19 & $(-34$ to -2$)$ \\
\hline Javiana & 1.1 & 99 & (57 to 153 ) & -7 & $(-26$ to 17$)$ \\
\hline | 4,[5],12:i:-** & 0.9 & 35 & $(-5$ to 74$)$ & 1 & $(-22$ to 29$)$ \\
\hline Muenchen & 0.4 & -13 & $(-35$ to 14$)$ & -4 & (-28 to 27$)$ \\
\hline Infantis & 0.3 & 60 & (19 to 113 ) & -20 & (-39 to 6) \\
\hline Montevideo & 0.3 & -30 & $(-47$ to -8$)$ & 24 & $(-7$ to 66$)$ \\
\hline Braenderup & 0.3 & 29 & $(-5$ to 76$)$ & 25 & (-8 to 70$)$ \\
\hline Saintpaul & 0.3 & -36 & $(-53$ to -14$)$ & -20 & $(-40$ to 9$)$ \\
\hline Thompson & 0.3 & 70 & (22 to 138$)$ & 32 & ( -5 to 84$)$ \\
\hline I 13,23:b:- ${ }^{\dagger \dagger}$ & 0.3 & $\mathrm{~N} / \mathrm{A}$ & N/A & $\mathrm{N} / \mathrm{A}$ & $\mathrm{N} / \mathrm{A}$ \\
\hline Heidelberg & 0.2 & -65 & $(-75$ to -52$)$ & -38 & $(-55$ to -15$)$ \\
\hline
\end{tabular}

Abbreviations: $\mathrm{Cl}$ = confidence interval; FoodNet = CDC Foodborne Diseases Active Surveillance Network; N/A = not applicable.

* Connecticut, Georgia, Maryland, Minnesota, New Mexico, Oregon, Tennessee, and selected counties in California, Colorado, and New York.

† Data for 2017 are preliminary.

$\S$ Per 100,000 population.

I Percentage change reported as increase or decrease.

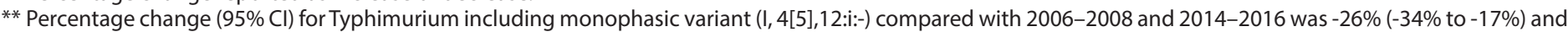
$-11 \%(-20 \%$ to $0 \%)$, respectively.

t† Comparisons could not be calculated for serotype I 13,23,b:l because of sparse data across the entire period.

specificity. Evaluations of panel tests have indicated high sensitivity and specificity, differing by test type and manufacturer. The Association of Public Health Laboratories recommends that clinical laboratories culture CIDT-positive specimens (9). The lack of isolates for $25 \%$ of bacterial infections in 2017 is cause for concern.

The findings in this report are subject to at least two limitations. First, the changing diagnostic landscape makes interpretation of incidence and trends difficult. In addition to actual increases in infection, increases in reported incidence might be due to some health care providers being more likely to order a CIDT because results are more quickly obtained than with traditional culture methods (1). Increases in incidence could also be due to increased use of DNA-based syndrome panel tests that diagnose pathogens not captured routinely by older methods. With improved sensitivity and specificity of DNAbased CIDTs, infections that previously would have remained undetected by culture methods might now be detected. Second, changes in incidence can reflect year-to-year variation rather than sustained trends.

Most foodborne illnesses can be prevented. New regulatory requirements aimed at reducing contamination of poultry meat might have contributed to decreases in incidence of infections caused by Salmonella serotypes Typhimurium and Heidelberg. Vaccination might also have contributed, but the extent of vaccination in poultry broiler flocks has not been reported. The declines in these and in STEC O157 infections provide supportive evidence that targeted control measures are effective. More control measures are needed and might be achieved with continued implementation of the FDA Food
Safety Modernization Act, ${ }^{\$ \subseteq}$ new or revised meat and poultry performance standards, and enhanced training and guidance for industry and inspection personnel. In particular, measures targeting specific Salmonella serotypes, including vaccination of broiler poultry flocks, might result in a marked decrease in human illness, as has been seen in the United Kingdom (10).

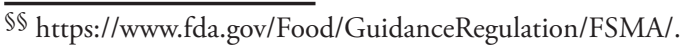

\section{Acknowledgments}

Workgroup members, Foodborne Diseases Active Surveillance Network (FoodNet), Emerging Infections Program, CDC; Brittany Behm, Staci Dixon, Elizabeth Greene, Logan Ray, Division of Foodborne, Waterborne, and Environmental Diseases, National Center for Emerging and Zoonotic Infectious Diseases, CDC; Neal Golden, Steven Mamber, and Joanna Zablotsky Kufel, U.S. Department of Agriculture's Food Safety and Inspection Service.

\section{Conflict of Interest}

No conflicts of interest were reported.

\footnotetext{
${ }^{1}$ Division of Foodborne, Waterborne, and Environmental Diseases, National Center for Emerging and Zoonotic Infectious Diseases, CDC; ${ }^{2}$ Oregon Health Authority; ${ }^{3}$ Tennessee Department of Health; ${ }^{4}$ Connecticut Department of Public Health; ${ }^{5}$ Colorado Department of Public Health and Environment; ${ }^{6}$ University of New Mexico, Albuquerque; ${ }^{7}$ New York State Department of Health; ${ }^{8}$ Maryland Department of Health; ${ }^{9}$ Minnesota Department of Health; ${ }^{10}$ Georgia Department of Public Health; ${ }^{11}$ California Department of Public Health; ${ }^{12}$ Food Safety and Inspection Service, U.S. Department of Agriculture, Atlanta, Georgia; ${ }^{13}$ Center for Food Safety and Applied Nutrition, Food and Drug Administration, Silver Spring, Maryland.

Corresponding author: Ellyn Marder, emarder1@cdc.gov, 404-718-4722.
} 


\section{Summary}

What is already known about this topic?

The incidence of infections transmitted commonly through food has remained largely unchanged for many years. Cultureindependent diagnostic tests (CIDTs) are increasingly used by clinical laboratories to detect enteric infections. CIDTs benefit public health surveillance by identifying illnesses caused by pathogens not captured routinely by previous laboratory methods.

What is added by this report?

Decreases in incidence of infection of Shiga toxin-producing Escherichia coli (STEC) 0157 and Salmonella serotypes Typhimurium and Heidelberg have been observed over the past 10 years. These declines parallel findings of decreased Salmonella contamination of poultry meat and decreased STEC 0157 contamination of ground beef.

What are the implications for public health practice?

As use of CIDTs continues to increase, higher, more accurate incidence rates might be observed. However, without isolates, public health laboratories are unable to subtype pathogens, determine antimicrobial susceptibility, and detect outbreaks. Further prevention measures are needed to decrease the incidence of infection by pathogens transmitted commonly through food.

\section{References}

1. Langley G, Besser J, Iwamoto M, et al. Effect of culture-independent diagnostic tests on future Emerging Infections Program surveillance. Emerg Infect Dis 2015;21:1582-8. https://doi.org/10.3201/ eid2109.150570
2. Geissler A, Huang J, Marder E, et al. The changing landscape of diagnostic testing for bacterial enteric pathogens and its impact on the surveillance and epidemiology of these pathogens-Foodborne Diseases Active Surveillance Network, USA, 2012-2015. Presented at the Council of State and Territorial Epidemiologists, Boise, Idaho; June 4-8, 2017.

3. Voetsch AC, Angulo FJ, Rabatsky-Ehr T, et al.; Emerging Infections Program FoodNet Working Group. Laboratory practices for stool specimen culture for bacterial pathogens, including Escherichia coli O157:H7, in the FoodNet sites, 1995-2000. Clin Infect Dis 2004;38:S190-7. https://doi.org/10.1086/381586

4. Dórea FC, Cole DJ, Hofacre C, et al. Effect of Salmonella vaccination of breeder chickens on contamination of broiler chicken carcasses in integrated poultry operations. Applied and Environmental Microbiology 2010;76:7820-5. https://doi.org/10.1128/AEM.01320-10

5. Clarkson LS, Tobin-D’Angelo M, Shuler C, et al. Sporadic Salmonella enterica serotype Javiana infections in Georgia and Tennessee: a hypothesis-generating study. Epidemiol Infect 2010;340-6. https://doi. org/10.1017/S0950268809990586

6. Mody RK, Luna-Gierke RE, Jones TF, et al. Infections in pediatric postdiarrheal hemolytic uremic syndrome: factors associated with identifying shiga toxin-producing Escherichia coli. Arch Pediatr Adolesc Med 2012;166:902-9. https://doi.org/10.1001/archpediatrics.2012.471

7. Khare R, Espy MJ, Cebelinski E, et al. Comparative evaluation of two commercial multiplex panels for detection of gastrointestinal pathogens by use of clinical stool specimens. J Clin Microbiol 2014;52:3667-73. https://doi.org/10.1128/JCM.01637-14

8. Murphy CN, Fowler RC, Iwen PC, Fey PD. Evaluation of the BioFire FilmArray ${ }^{\bullet}$ gastrointestinal panel in a Midwestern academic hospital. Eur J Clin Microbiol Infect Dis 2017;36:747-54. https://doi. org/10.1007/s10096-016-2858-7

9. Shea S, Kubota KA, Maguire H, et al. Clinical microbiology laboratories' adoption of culture-independent diagnostic tests is a threat to foodbornedisease surveillance in the United States. J Clin Microbiol 2017;55:10-9. https://doi.org/10.1128/JCM.01624-16

10. O'Brien SJ. The "decline and fall" of nontyphoidal salmonella in the United kingdom. Clin Infect Dis 2013;56:705-10. https://doi. org/10.1093/cid/cis967 